\title{
Three-layer Non-hydrostatic Staggered Scheme for Free Surface Flow
}

\author{
Ade C. Bayu*, S. R. Pudjaprasetya and I. Magdalena \\ Industrial \& Financial Mathematics Research Group, \\ Faculty of Mathematics \& Natural Sciences, \\ Institut Teknologi Bandung, \\ Jalan Ganesha 10, Bandung, 40132, Indonesia
}

\begin{abstract}
In this paper, a finite difference algorithm using a three-layer approximation for the vertical flow region to solve the 2D Euler equations was considered. In this algorithm, the pressure was split into hydrostatic and hydrodynamic parts, and the predictor-corrector procedure was applied. In the predictor step, the momentum hydrostatic model was formulated. In the corrector step, the hydrodynamic pressure was accommodated after solving the Laplace equation using the Successive Over Relaxation (SOR) iteration method. The resulting algorithm was first tested to simulate a standing wave over an intermediate constant depth. Dispersion relation of the scheme was derived and it was shown to agree with the analytical dispersion relation for $k d<\pi$ with $94 \%$ accuracy. The second test case was a solitary wave simulation. Our computed solitary wave propagated with constant velocity, undisturbed in shape, and confirmed the analytical solitary wave. Finally, the scheme was tested to simulate the appearance of the undular bore. The result shows a good agreement with the result from the finite volume scheme for the Boussinesq-type model by Soares-Frazão and Guinot (2008).
\end{abstract}

AMS subject classifications: 65M10, 78A48

Key words: the 2D Euler equations, non-hydrostatic scheme, solitary wave, undular bore

\section{Introduction}

Free surface flows are mostly described using the shallow water equations (SWE) with hydrostatic pressure distribution and depth-averaged velocity assumptions. With those assumptions, the SWE cannot be used to describe the secondary wave phenomena, such as an undular bore. Undular bore is a dispersive wave phenomena observed in nature. Undular bores and shocks generated by dam-break flows or tsunamis are examined by Kim and Lynett [1] using the 2D Euler equations that account for the hydrodynamic pressure term.

*Corresponding author. Email addresses: ade_zildji@yahoo.co.id (Ade C. Bayu), sr_pudjap@math.itb.ac.id (S. R. Pudjaprasetya), ikha.magdalena@math.itb.ac.id (I. Magdalena) 
This paper aims to develop a numerical model to simulate water wave dynamics that involve dispersion effects. Here, we consider the 2D Euler equations applicable to a column of water, assumed as an inviscid ideal fluid of constant temperature. Since we are dealing with free surface flows, an extra equation for free surface condition is needed, and that comes from a depth integrated continuity equation after incorporating the kinematic boundary equation. For the sake of numerical computation efficiency, the vertical axis on the Euler equations is resolved only by a few cells. In this paper, we focus on the three layer approximation of the 2D Euler model.

The organization of this paper is as follows. The first two sections discusses the governing equations and the formulation of the three-layer staggered grid scheme. Discussion is followed with the numerical dispersion relation and its comparison with the analytical dispersion relation. In Section 4 the scheme is validated with three benchmark tests: standing wave, solitary wave, and tidal bore with undulations. Those are test cases for the non-hydrostatic numerical schemes. Conclusions are outlined in the last section.

\section{The Governing Equations}

We consider a physical domain that is bounded on top by the free surface $z=\eta(x, t)$ and the bottom $z=-d(x)$. The 2D Euler equations for free surface flows in $x-$ and $z$-direction with velocity components $u$ and $w$ as the dependent variables are given by the momentum and continuity equations as noted in [4], i.e.

$$
\begin{aligned}
\frac{\partial u}{\partial t}+u \frac{\partial u}{\partial x}+w \frac{\partial u}{\partial z}+\frac{1}{\rho} \frac{\partial p}{\partial x} & =0 \\
\frac{\partial w}{\partial t}+u \frac{\partial w}{\partial x}+w \frac{\partial w}{\partial z}+\frac{1}{\rho} \frac{\partial p}{\partial z} & =0 \\
\frac{\partial u}{\partial x}+\frac{\partial w}{\partial z} & =0
\end{aligned}
$$

where $p(z, x, t)$ is the pressure, $\rho$ the fluid density, and $g$ the acceleration due to gravity. For horizontally dominant flow, in which the vertical velocity is high order, then (2.1), (2.2), and (2.3) can be simplified to

$$
\begin{aligned}
\frac{\partial u}{\partial t}+u \frac{\partial u}{\partial x}+\frac{1}{\rho} \frac{\partial p}{\partial x} & =0 \\
\frac{\partial w}{\partial t}+\frac{1}{\rho} \frac{\partial p}{\partial z} & =0 \\
\frac{\partial u}{\partial x}+\frac{\partial w}{\partial z} & =0 .
\end{aligned}
$$

Since we are examining the free surface flows, we need an additional equation for determining water level $\eta(x, t)$. The equation is obtained by integrating the continuity 


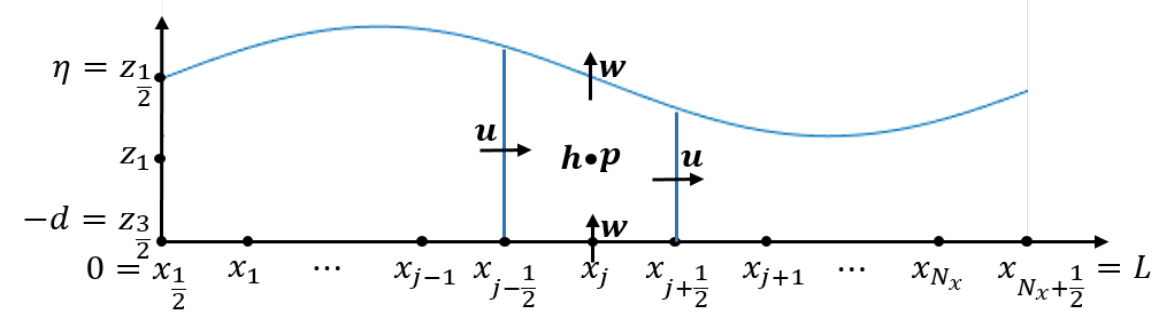

Figure 1: A One-layer staggered grid and arrangement of unknowns in a cell of mass.

equation (2.6) over the water depth $h=\eta+d$, i.e.

$$
\int_{-d}^{\eta}\left(\frac{\partial u}{\partial x}+\frac{\partial w}{\partial z}\right) d z=0
$$

Then subsequent use of the Leibniz's rule and insertion of the kinematic boundary conditions, thus obtained the equation for calculating $h$ (we can easily obtain $\eta$ from $h$ ) explicitly as follows

$$
\frac{\partial h}{\partial t}=-\frac{\partial(h u)}{\partial x}
$$

\section{Numerical Methods}

In this section, we describe the numerical method for a three-layer non-hydrostatic scheme. Consider a spatial domain $\Omega=\{(z, x) \mid-d \leq z \leq \eta, 0 \leq x \leq L\}$. The domain is partitioned into a staggered grid. On a staggered grid, vector variables $(u$ and $w)$ are located in the half grid, while scalar variables ( $h$ and $p$ ) are located at the full grid. Staggered grid for one-layer model is shown in Figure 1.

Figure 1 shows a mass cell centered at $\left(z_{1}, x_{j}\right)$, bounded by $\eta(x, t)$ on top and $-d(x)$ on the bottom and the vertical grid lines $x_{j \pm \frac{1}{2}}$. The width $\Delta x$ is fixed, but the layer thickness $\Delta z$ changes every time iteration and equals to $h(x, t)$. We consider a three-layer for this scheme. Previously we have tried two-layer approximation, but the result is still not good enough (we show the result in Section 4). We partitioned $-d \leq z \leq \eta$ into three layers with interval width $\Delta z=h(x, t) / 3$. Staggered grid for the three-layer model is shown in Figure 2, see [2] for reference.

When variables in Figure 2 are written with their corresponding indexes, they read as

$$
\begin{aligned}
h\left(x_{j}, t_{n}\right) & \equiv h_{j}^{n}, \\
p\left(z_{i}, x_{j}, t_{n}\right) & \equiv p_{i, j}^{n}, \\
u\left(z_{i}, x_{j+\frac{1}{2}}, t_{n}\right) & \equiv u_{i, j+\frac{1}{2}}^{n}, \\
w\left(z_{i+\frac{1}{2}}, x_{j}, t_{n}\right) & \equiv w_{i+\frac{1}{2}, j}^{n} .
\end{aligned}
$$




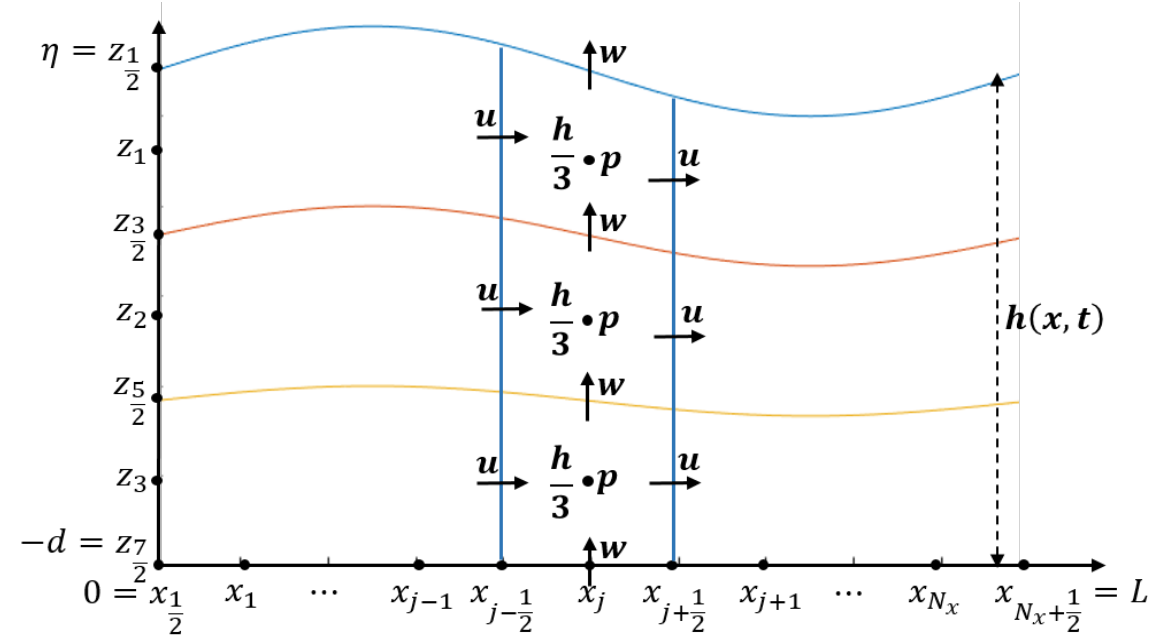

Figure 2: A Three-layer staggered grid and arrangement of unknowns in a column of mass.

Consistent discretization of (2.8) and (2.4-2.6) are as follows

$$
\begin{aligned}
& \frac{h_{j}^{n+1}-h_{j}^{n}}{\Delta t}=-\frac{(h u)_{j+\frac{1}{2}}^{n}-(h u)_{j-\frac{1}{2}}^{n}}{\Delta x} \text { for } j=1,2, \ldots, N_{x}, \\
& \frac{u_{i, j-\frac{1}{2}}^{n+1}-u_{i, j-\frac{1}{2}}^{n}}{\Delta t}+u u_{x_{i, j-\frac{1}{2}}^{n}}^{n}+\frac{p_{i, j}^{n+1}-p_{i, j-1}^{n+1}}{\rho \Delta x}=0 \text { for } i=1,2,3 ; j=1,2, \ldots, N_{x}+1, \\
& \frac{w_{i-\frac{1}{2}, j}^{n+1}-w_{i-\frac{1}{2}, j}^{n}}{\Delta t}+\frac{p_{i-1, j}^{n+1}-p_{i, j}^{n+1}}{\rho \Delta z}=0 \text { for } i=1,2,3,4 ; j=1,2, \ldots, N_{x}, \\
& \frac{u_{i, j+\frac{1}{2}}^{n+1}-u_{i, j-\frac{1}{2}}^{n+1}}{\Delta x}+\frac{w_{i-\frac{1}{2}, j}^{n+1}-w_{i+\frac{1}{2}, j}^{n+1}}{\Delta z}=0 \text { for } i=1,2,3 ; j=1,2, \ldots, N_{x},
\end{aligned}
$$

where

$$
(h u)_{j+\frac{1}{2}}^{n}=\sum_{i=1}^{3}{\frac{}{*} h_{j+\frac{1}{2}}^{n}}_{3}^{n} u_{i, j+\frac{1}{2}} .
$$

Variable $h_{j}^{n}$ does not lie in the half grid so that it is denoted as ${ }^{*} h_{j}^{n}$ and the value is approximated by first-order upwind method as in [5], i.e.

$$
* h_{j+\frac{1}{2}}^{n}= \begin{cases}h_{j}^{n} & \text { if } u_{i, j+\frac{1}{2}}^{n}>0 \\ h_{j+1}^{n} & \text { if } u_{i, j+\frac{1}{2}}^{n}<0 \\ \max \left(h_{j}^{n}, h_{j+1}^{n}\right) & \text { if } u_{i, j+\frac{1}{2}}^{n}=0\end{cases}
$$


The non-linear term in (3.2) is approximated by the momentum conservative scheme as in $[5]$.

We split the pressure $p$ into hydrostatic part and hydrodynamic part as follows

$$
p(z, x, t)=\rho g h(x, t)+\Delta q(z, x, t)
$$

with $\Delta q$ denotes the hydrodynamic pressure and $q=\rho g h(x, t)$ denotes the hydrostatic pressure. Then the numerical procedure for solving equations (3.2) and (3.3) is formulated in two steps: the predictor and corrector. Predictor step is to calculate the velocity $u$ and $w$ using the hydrostatic pressure $q$ using

$$
\begin{aligned}
& u_{i, j-\frac{1}{2}}^{*}=u_{i, j-\frac{1}{2}}^{n}-\Delta t u u_{x_{i, j-\frac{1}{2}}^{n}}-\frac{g \Delta t}{3 \Delta x}\left(h_{j}^{n+1}-h_{j-1}^{n+1}\right), \\
& w_{i-\frac{1}{2}, j}^{*}=w_{i-\frac{1}{2}, j}^{n}-\frac{g \Delta t}{3 \Delta z}\left(h_{j}^{n+1}-h_{j}^{n+1}\right)=w_{i-\frac{1}{2}, j}^{n} .
\end{aligned}
$$

Corrector step is updating the predicted value velocity $\mathrm{u}$ and $\mathrm{w}$ using the hydrodynamic term $\Delta q$. The corrector step is

$$
\begin{aligned}
& u_{i, j-\frac{1}{2}}^{n+1}=u_{i, j-\frac{1}{2}}^{*}-\frac{\Delta t}{\rho \Delta x}\left(\Delta q_{i, j}^{n+1}-\Delta q_{i, j-1}^{n+1}\right), \\
& w_{i-\frac{1}{2}, j}^{n+1}=w_{i-\frac{1}{2}, j}^{*}-\frac{\Delta t}{\rho \Delta z}\left(\Delta q_{i-1, j}^{n+1}-\Delta q_{i, j}^{n+1}\right) .
\end{aligned}
$$

\section{Non-hydrostatic Solver}

Non-hydrostatic pressure is a major issue in this paper. We use the hydrodynamic pressure term $\Delta q$ in the corrector step to update the predicted values, so $\Delta q$ should be computed first. A way to do that will be described in the following. Substituting (3.10) and (3.11) into (3.4) followed by multiplication with $\Delta x$ and $\Delta z$ to yields

$$
a_{1}\left(\Delta q_{i, j+1}^{n+1}+\Delta q_{i, j-1}^{n+1}\right)+a_{2}\left(\Delta q_{i-1, j}^{n+1}+\Delta q_{i+1, j}^{n+1}\right)-a_{0} \Delta q_{i, j}^{n+1}=q_{i, j}^{*},
$$

where $a_{1}=\frac{\Delta z}{\Delta x}, a_{2}=\frac{\Delta x}{\Delta z}$, and $a_{0}=2\left(a_{1}+a_{2}\right)$. The right-hand side of equation (3.12) reads

$$
q_{i, j}^{*}=\frac{\rho}{\Delta t}\left[\left(u_{i, j+\frac{1}{2}}^{*}-u_{i, j-\frac{1}{2}}^{*}\right) \Delta z+\left(w_{i-\frac{1}{2}, j}^{*}-w_{i+\frac{1}{2}, j}^{*}\right) \Delta x\right] .
$$

Equation (3.12) is a Poisson equation in variable $\Delta q$, which is solved using the successive over relaxation (SOR) iteration method. In SOR, starting with the initial $\Delta q_{i, j}^{0}$ as in the initial guess, we compute iteratively $\Delta q_{i, j}^{r+1}$, for $r=0,1,2, \ldots$ using the following equation

$$
\Delta q_{i, j}^{r+1}=(1-\Omega) \Delta q_{i, j}^{r}+\frac{\Omega}{a_{0}}\left[a_{1}\left(\Delta q_{i, j+1}^{r}+\Delta q_{i, j-1}^{r+1}\right)+a_{2}\left(\Delta q_{i-1, j}^{r+1}+\Delta q_{i+1, j}^{r}\right)-q_{i, j}^{*}\right],
$$


in which the parameter $\Omega=1.4$ is used. The above iteration is repeated until the solution converges, i.e. $\left|\Delta q_{i, j}^{r+1}-\Delta q_{i, j}^{r}\right|<\epsilon$ where $\epsilon$ is the tolerance, here taken to be $10^{-5}$. The SOR iteration results $\left(\Delta q_{i, j}^{n+1}\right)$ is then used to update the velocity $u_{i, j-\frac{1}{2}}^{n+1}, w_{i-\frac{1}{2}, j}^{n+1}$ using (3.10) and (3.10).

The computational steps of the non-hydrostatic scheme are as follows

1. Compute water depth $h^{n+1}$ using equation (3.1).

2. Compute the predicted velocities $u^{*}$ and $w^{*}$ using equations (3.8) and (3.9).

3. Compute $q^{*}$ using equation (3.13).

4. Compute the hydrodynamic pressure $\Delta q^{n+1}$ by solving equation (3.14) with the SOR iteration method.

5. Using computed $\Delta q^{n+1}$, the corrected velocities $u^{n+1}$ and $w^{n+1}$ are computed.

Note that step 1-2 are the hydrostatic scheme.

\section{Numerical Simulations and Validation}

Water waves propagate in the open sea, they undergo dispersion effects. Dispersion means that waves with different wave numbers propagate with different phase speeds. Propagation of each monochromatic wave is determined by dispersion relation of the system. Airy theory of water waves yields the analytical dispersion

$$
\omega^{2}=g k \tanh (k d)
$$

where $\omega$ is wave frequency and $k$ is wave number.

The numerical scheme proposed here is expected to have a dispersion relation that is comparable with the analytical dispersion (4.1). The numerical dispersion relation can be obtained by substituting the following wave like solutions:

$$
\left\{\begin{array}{l}
u_{i, j+\frac{1}{2}}^{n} \equiv u_{i} e^{I\left(k\left(j+\frac{1}{2}\right) \Delta x-\omega n \Delta t\right)} \\
w_{i-\frac{1}{2}, j}^{n} \equiv w_{i-\frac{1}{2}} e^{I(k j \Delta x-\omega n \Delta t)} \\
p_{i, j}^{n} \equiv p_{i} e^{I(k j \Delta x-\omega n \Delta t)} \\
h_{j}^{n} \equiv h e^{I(k j \Delta x-\omega n \Delta t)}
\end{array}\right.
$$

with $I^{2}=-1$, into the linearized form of (3.1-3.4). Detailed derivation is given in the Appendix, and the numerical dispersion relation itself reads

$$
\omega_{n u m}^{2}=\frac{g d k^{2}\left(6(k d)^{4}+216(k d)^{2}+1458\right)}{(k d)^{6}+54(k d)^{4}+729(k d)^{2}+1458} .
$$




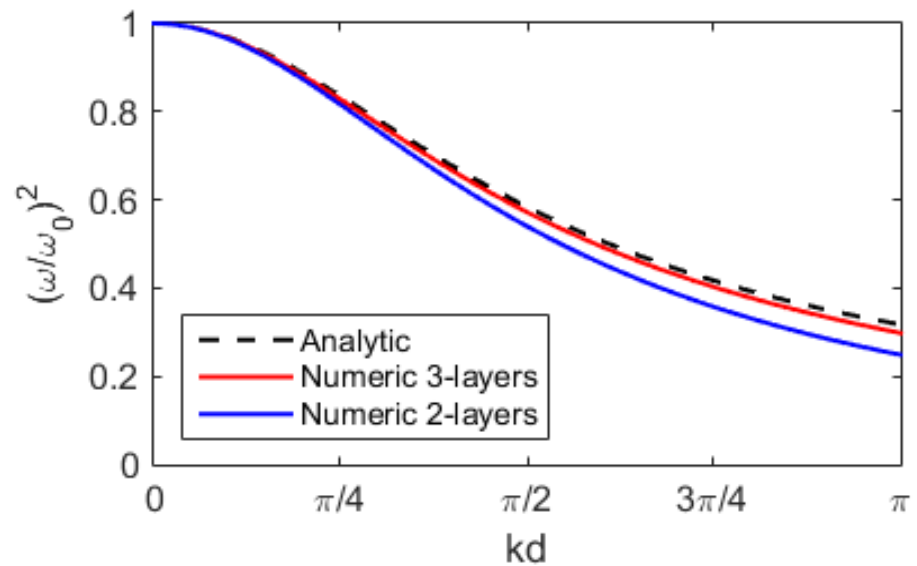

Figure 3: The numerical dispersion $\left(\omega_{\text {num }} / \omega_{0}\right)^{2}$ in comparison with the analytic dispersion curves are plotted using $d=10 \mathrm{~m}$ and $g=9.8 \mathrm{~m} / \mathrm{s}^{2}$.

In Figure 3 we plot the numerical and analytical dispersion relations $\left(\omega / \omega_{0}\right)^{2}$, with $\omega_{0}^{2}=g d k^{2}$. The numerical dispersion relation of the proposed method developed using a three-layer of the fluids can approximate the analytical dispersion relation quite well for $k d<\pi$ with 94\% accuracy. Different result shown if the proposed method developed using two-layer of fluids. It has a large error although for $k d<\pi$.

The numerical scheme have been evaluated by considering three test cases of free surface flows. The first test case is standing wave in a closed basin, which test the ability of the numerical scheme in simulating monochromatic wave motion with the correct frequency. The second test case is the solitary wave propagation. The last test case is the undular bore propagation. Those tests aimed to verify the non-hydrostatic effects of the numerical scheme through comparison with analytical solutions or experimental results.

\subsection{Standing Wave in a Closed Basin}

Standing wave can be defined as a combination of two waves propagate in the opposite directions, each of them has the same frequency and amplitude. Results of this combination is a wave which only moves up and down with constant amplitude. Here, we write quantities of measurements without units by convention that SI units are used. We consider a standing wave in a closed basin with length $L$, constant depth $d=10$, and initial conditions

$$
\left\{\begin{array}{l}
\eta(x, 0)=A \cos (k x), \quad 0 \leq x \leq L \text { with } L=\frac{2 \pi}{k}, \\
u(z, x, 0)=0, \quad w(z, x, 0)=0,
\end{array}\right.
$$

where $A$ is the amplitude of standing wave and $k$ is the wave number. Boundary conditions are $u(z, 0, t)=0$ and $u(z, L, t)=0$. Here, the wavelength equals to the length of the basin. 


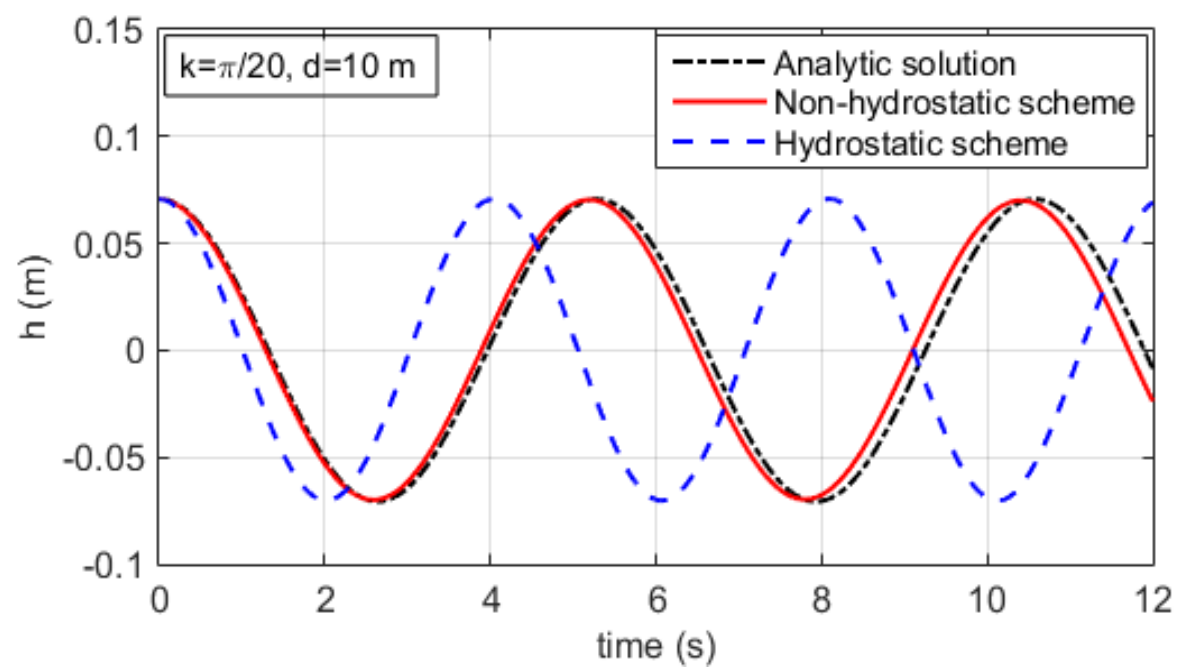

Figure 4: Wave signal calculated using hydrostatic scheme, non-hydrostatic scheme and the analytical surface elevation, recorded at $x=35 \mathrm{~m}$.

The simulation results are compared with the analytical solution. The analytical solution of initial conditions (4.4) is the sum of two wave components propagate in the opposite direction, i.e.

$$
\eta(x, t)=\frac{A}{2}[\cos (k x-\omega t)+\cos (k x+\omega t)], \text { with } \omega=\sqrt{g k \tanh (k d)} .
$$

Using parameters $A=0.1, d=10$, and $\Delta x=0.1$, and $\Delta t=0.01$, two standing wave simulations were performed. The first wave uses $k=\frac{\pi}{20}$ or $k d=\frac{\pi}{2}$. The resulting wave signal recorded at $x=35$ is plotted in Figure 4 together with the the analytical wave (4.5). It is shown that the three layer non-hydrostatic scheme produces wave signal with frequency 0.192 that approximates the analytical frequency 0.189 , whereas wave signal resulting from the hydrostatic scheme has frequency 0.247 which has a much larger error. The second simulation uses $k=\frac{\pi}{10}$, or $k d=\pi$, which is at the boundary between intermediate and deep water area. The resulting wave signal recorded at $x=17.5$ is plotted in Figure 5 together with the analytical wave. This comparison show that our three layer non-hydrostatic scheme computes wave signal with an accurate frequency.

\subsection{Solitary Wave Propagation}

The second test case is the propagation of a solitary wave. A dispersive nonlinear model of water waves admits a solitary wave solution. The analytical formula for a solitary wave 


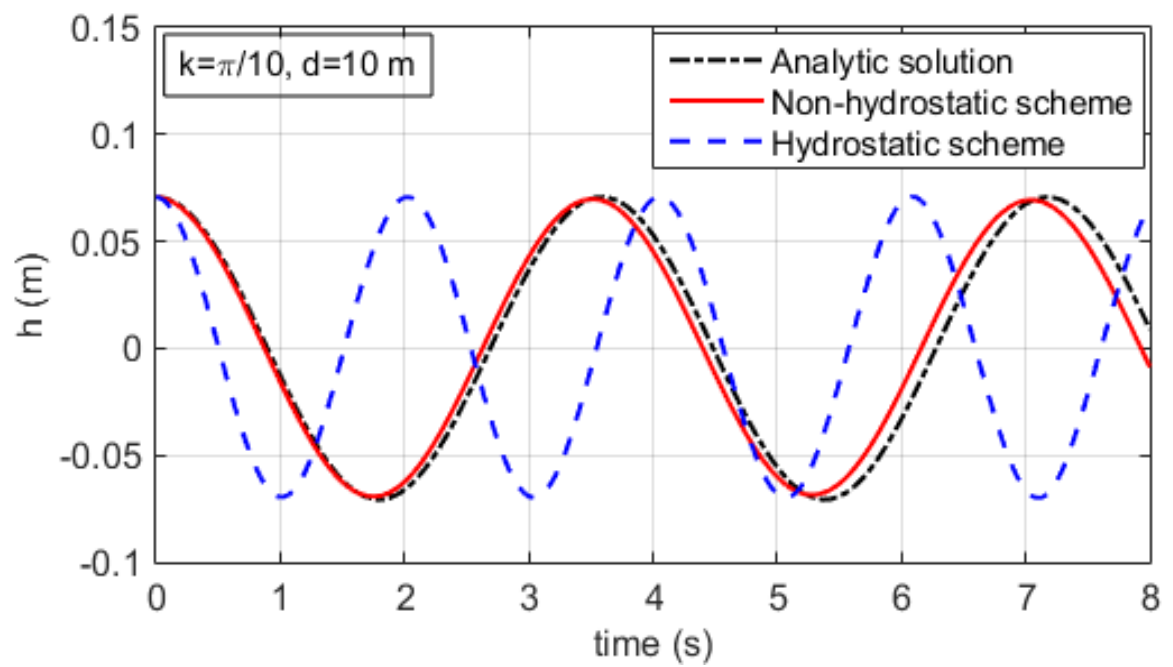

Figure 5: Wave signal calculated using hydrostatic scheme, non-hydrostatic scheme and the analytical surface elevation, recorded at $x=17.5 \mathrm{~m}$.

with amplitude $A$ reads

$$
\eta(x, t)=A \operatorname{sech}^{2}\left[\sqrt{\frac{3 A}{4 d^{2}(d+A)}}\left(x-x_{0}-c t\right)\right] .
$$

This is a wave of permanent form that propagates to the right undisturbed in shape with phase velocity $c=\sqrt{g(d+A)}$, with $d$ is the constant water depth.

Our aim is to simulate the right running solitary wave using our numerical scheme of Euler equations. Since our scheme is a model for both left and right running waves, therefore in the computation we take an initial solitary wave with doubled amplitude $2 A$, and let it falls with zero velocity, so that it splits into two identical waves. When we follow the right running wave, it is the solitary wave of amplitude $A$ comparable to (4.6). To be precise the following initial conditions is used in the computation:

$$
\left\{\begin{array}{l}
\eta(x, 0)=2 A \operatorname{sech}^{2}\left[\sqrt{\frac{3 A}{4 d^{2}(d+A)}}\left(x-x_{0}\right)\right] ;-L \leq x \leq L, \\
u(z, x, 0)=0 ; \quad w(z, x, 0)=0
\end{array}\right.
$$

with parameters $A=1, d=10, x_{0}=0, \Delta x=1, \Delta t=0.05$, and $L=3000$. The boundary conditions are $u(z,-L, t)=0$ and $u(z,-L, t)=0$. Figure 6 shows that the numerical solitary wave preserves its shape and in a good agreement with the analytical solitary wave. The numerical solitary wave is still good for along run. Figure 7 shows that the wave height decreased slightly, but its shape is still preserved. 


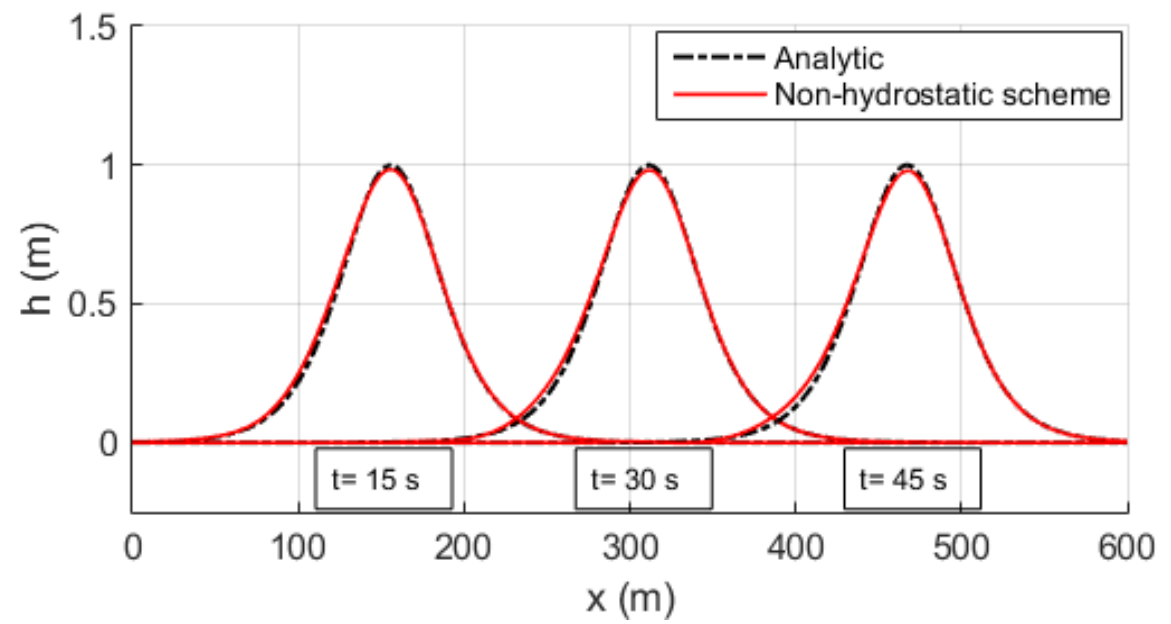

Figure 6: Comparison between computed and analytical solitary wave at $t=15,30$, and $45 \mathrm{~s}$.

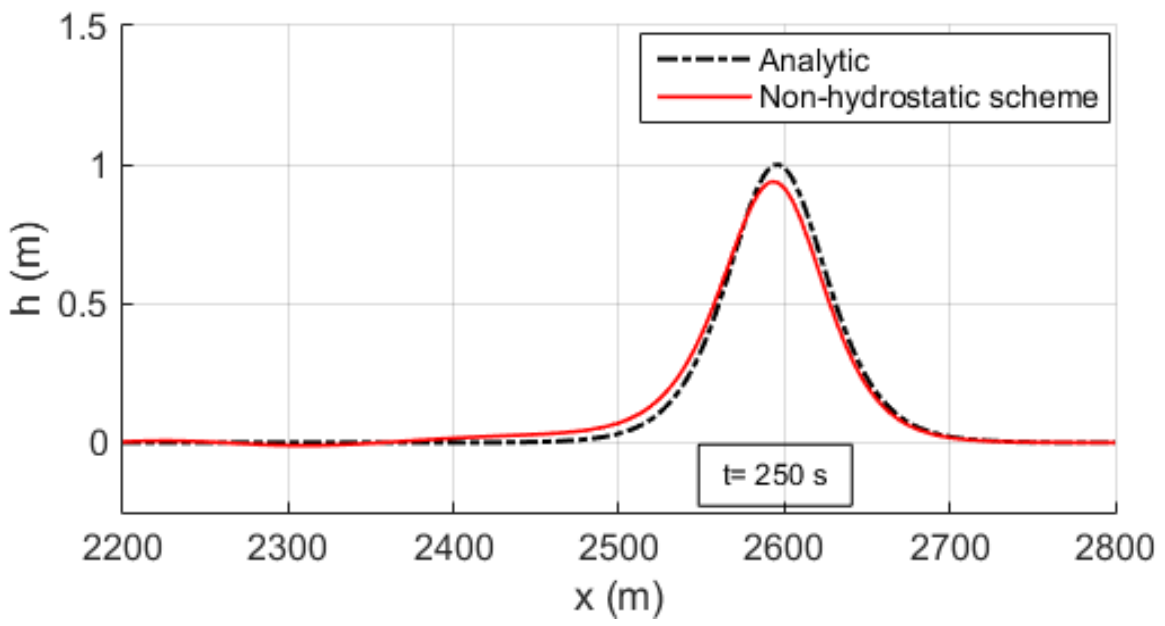

Figure 7: Comparison between computed and analytical solitary wave for long run after $250 \mathrm{~s}$. 


\subsection{Undular Bore Propagation}

Undular bore is a positive surges usually observed in shallow water area with the advancing front, sometimes occupied with a train of well-defined free-surface undulations. The undulations form a standing wave pattern but the amplitude was enlarged. Numerical simulation of undular bore needs a model that incorporate hydrodynamic pressure term. Here, we adopt the benchmark test of undular bore by [6]. For that purpose the following initial conditions are used

$$
\left\{\begin{array}{l}
u(z, x, 0)=\frac{1}{2} u_{0} \sqrt{g d}\left[1-\tanh \left(\frac{x}{a}\right)\right] ;-10 \leq x \leq 250 \\
\eta(x, 0)=\frac{1}{g}\left(u(z, x, 0) \sqrt{g d}+\frac{1}{4} u^{2}(z, x, 0)\right) \\
w(z, x, 0)=0
\end{array}\right.
$$

with parameter $a$ associated with the wave steepness, where smaller $a$ leads to a steeper wave. For computation we use the following parameters $a=5, d=1, u_{0}=0.1$, with $\Delta x=0.2$ and $\Delta t=0.05$. The boundary conditions are $u(z,-10, t)=0$ and $u(z, 250, t)=$ 0 .

Surface wave profiles at subsequent times are presented in Figure 8, and around $t=$ 9 undulations start to develop. As time progresses, the undulations grow in numbers and in amplitudes. In Figure 9, the early stages of undular bore development resulting from our three layer non-hydrostatic scheme, are plotted together with those from SoaresFrazão in [6] computed using the MUSCL4 scheme. It is shown that our results are nicely comparable, also the bore propagates with an accurate speed. This undular bore of SoaresFrazão in [6] is known to be the main benchmark test for undular bore, and in [7] they have been validated with experimental data.

\section{Conclusion}

The three-layer non-hydrostatic staggered scheme has been successfully implemented to solve the 2D Euler equations, and applied for free surface flows. Dispersion relation of the scheme was derived and shown to conform the analytical dispersion relation. Standing wave simulation has shown to achieve an accurate frequency, including those for rather

short waves. Solitary wave simulation produces a wave of permanent form propagating with constant velocity, in a good agreement with the analytical solitary wave. Moreover, our simulation can demonstrate the development of undulations near the tidal bore front, that agrees well with those results from MUSCL4 scheme. Good agreement with various benchmark tests have shown that the scheme is suitable for free surface simulations with non-linearity and dispersion. 

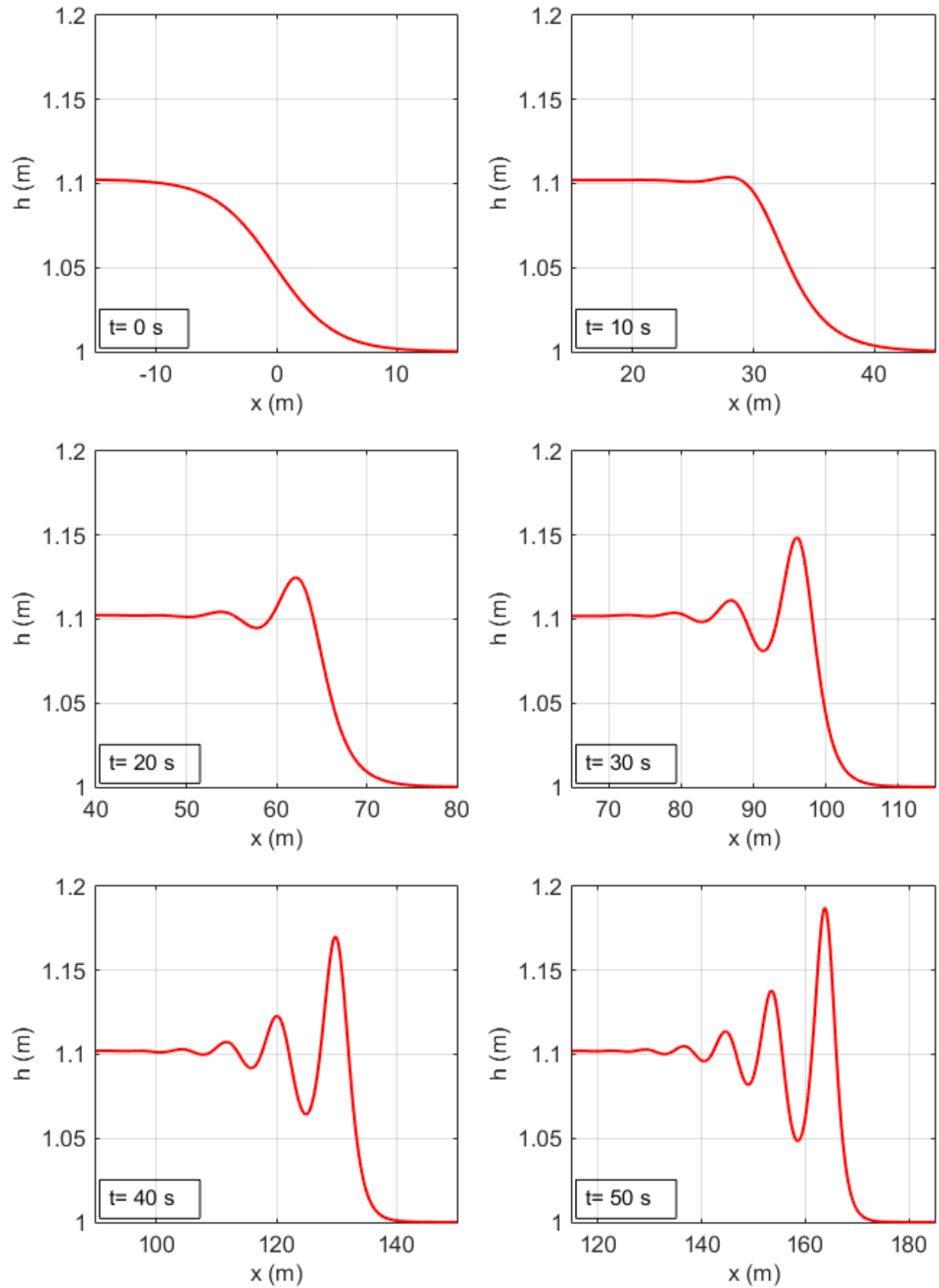

Figure 8: The Appearance of undular bore propagation computed using non-hydrostatic numerical scheme. 

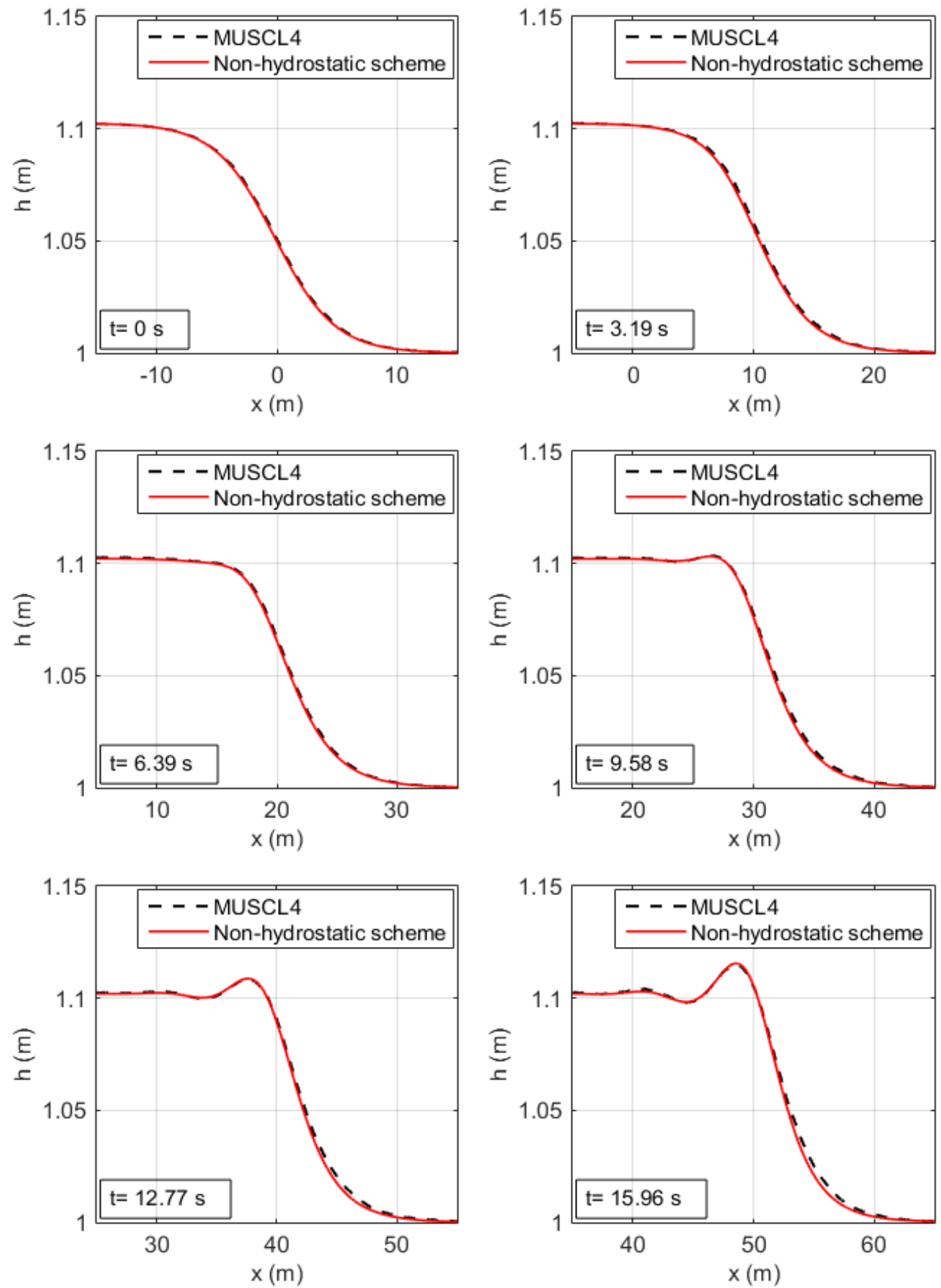

Figure 9: Comparison the appearance of undular bore propagation between results from three-layer non-hydrostatic numerical scheme and MUSCL4 scheme. 


\section{Acknowledgments}

We thank the anonymous referees for useful comments that have improved the presentation of this paper. This research was supported by Institut Teknologi Bandung research grant 107a/I1.C01/PL/2017 and 1081/11.C01/PL/2017.

\section{References}

[1] D. H. Kim And P. J. LynetT, Dispersive and nonhydrostatic pressure effects at the front of surge, J. Hydraul. Eng., 137(2011), pp. 754-765.

[2] J. KÄMPF, Advanced Ocean Modelling Using Open-Source Software, Springer-Verlag, Berlin Heidelbert, 2010.

[3] G. Stelling and M. ZiJlema, An accurate and efficient finite-difference algorithm for nonhydrostatic free surface flow with application to wave propagation, Int. J. Numer. Meth. Fluids, 43(2003), pp. 1-23.

[4] J. K. Hunter, An introduction to the incompressible Euler equations [Lecture note], 2006, Retrieved from www.math.ucdavis.edu/ hunter/notes/euler.pdf.

[5] G. Stelling And S. P. A. Duinmeiver, A staggered conservative scheme for every Froude number in rapidly varied shallow water flows, Int. J. Numer. Meth. Fluids, 43(2003), pp. 1329-1354.

[6] S. Soares-FrazÃo And V. Guinot, A second-order semi-implicit hybrid scheme for onedimensional Boussinesq-type waves in rectangular channels, Int. J. Numer. Meth. Fluids, 58(2008), pp. 237-261.

[7] S. SOARES-FrAZÃO AND Y. ZECH, Undular bores and secondary waves-experiments and hybrid finite-volume modeling, J. Hydr. Research, 40(2002), pp. 33-43. 
Three-layer Non-hydrostatic Staggered Scheme for Free Surface Flow

\section{Appendix}

Here, we give detail derivation of dispersion relation (4.3) of the three-layer nonhydrostatic scheme. We start with the linearized form of (3.1-3.4), i.e.

$$
\begin{aligned}
& \frac{\eta_{j}^{n+1}-\eta_{j}^{n}}{\Delta t}+\frac{(h u)_{j+\frac{1}{2}}^{n}-(h u)_{j-\frac{1}{2}}^{n}}{\Delta x}=0, \\
& \frac{u_{i, j+\frac{1}{2}}^{n+1}-u_{i, j+\frac{1}{2}}^{n}}{\Delta t}+\frac{p_{i, j+1}^{n+1}-p_{i, j}^{n+1}}{\rho \Delta x}=0, \\
& \frac{w_{i+\frac{1}{2}, j}^{n+1}-w_{i+\frac{1}{2}, j}^{n}}{\Delta t}+\frac{p_{i, j}^{n+1}-p_{i+1, j}^{n+1}}{\rho \Delta z}=0, \\
& \frac{u_{i, j+\frac{1}{2}}^{n+1}-u_{i, j-\frac{1}{2}}^{n+1}}{\Delta x}+\frac{w_{i-\frac{1}{2}, j}^{n+1}-w_{i+\frac{1}{2}, j}^{n+1}}{\Delta z}=0,
\end{aligned}
$$

where $(h u)_{j+\frac{1}{2}}^{n}=\sum_{i=1}^{3} \frac{{ }^{*} h_{j+\frac{1}{2}}^{n}}{3} u_{i, j+\frac{1}{2}}^{n}, \Delta z=\frac{h_{j}^{n}}{3}$ and assume $h_{j}^{n}=d$, so (5.1) can be rewritten as follows

$$
\frac{h_{j}^{n+1}-h_{j}^{n}}{\Delta t}+\frac{d\left(\left(u_{1, j+\frac{1}{2}}^{n}-u_{1, j-\frac{1}{2}}^{n}\right)+\left(u_{2, j+\frac{1}{2}}^{n}-u_{2, j-\frac{1}{2}}^{n}\right)+\left(u_{3,1, j+\frac{1}{2}}^{n}-u_{3, j-\frac{1}{2}}^{n}\right)\right)}{3 \Delta x}=0,
$$

Subtitute (4.2) to the equations system (5.2-5.5), obtained

$$
\begin{cases}\frac{1}{\Delta t}\left(e^{-I \omega \frac{\Delta t}{2}}-e^{I \omega \frac{\Delta t}{2}}\right) \eta+\frac{d e^{I \omega \frac{\Delta t}{2}}}{3 \Delta x}\left(e^{I k \frac{\Delta x}{2}}-e^{-I k \frac{\Delta x}{2}}\right)\left(u_{1}+u_{2}+u_{3}\right) & =0 \\ \frac{1}{\Delta t}\left(e^{-I \omega \frac{\Delta t}{2}}-e^{I \omega \frac{\Delta t}{2}}\right) u_{i}+\frac{e^{-I \omega \frac{\Delta t}{2}}}{\rho \Delta x}\left(e^{I k \frac{\Delta x}{2}}-e^{-I k \frac{\Delta x}{2}}\right) p_{i} & =0 ; i=1,2,3 \\ \frac{1}{\Delta t}\left(e^{-I \omega \frac{\Delta t}{2}}-e^{I \omega \frac{\Delta t}{2}}\right) w_{\frac{1}{2}}+\frac{6 e^{-I \omega \frac{\Delta t}{2}}}{\rho d}\left(p_{0}-p_{1}\right) & =0 \\ \frac{1}{\Delta t}\left(e^{-I \omega \frac{\Delta t}{2}}-e^{I \omega \frac{\Delta t}{2}}\right) w_{i+\frac{1}{2}}+\frac{3 e^{-I \omega \frac{\Delta t}{2}}}{\rho d}\left(p_{i}-p_{i+1}\right) & =0 ; i=1,2 \\ \frac{1}{\Delta x}\left(e^{I k \frac{\Delta x}{2}}-e^{-I k \frac{\Delta x}{2}}\right) u_{i}+\frac{3}{d}\left(w_{i-\frac{1}{2}}-w_{i+\frac{1}{2}}\right) & =0 ; i=1,2,3\end{cases}
$$

Variable $p_{0}$ declared the pressure in $z=\frac{1}{2}$, so it is $p\left(\frac{1}{2}, x, t\right)=\rho g \eta(x, t)$. The distance between $p\left(\frac{1}{2}, x, t\right)$ and $p(1, x, t)$ is $\Delta z / 2$, so that $\frac{1}{\Delta z / 2}=\frac{6}{d}$ appears in the 5 th equation of system above. 
Euler formula gives $e^{-I \omega \frac{\Delta t}{2}}-e^{I \omega \frac{\Delta t}{2}}=-2 I \sin \frac{\omega \Delta t}{2}, e^{I k \frac{\Delta x}{2}}-e^{-I k \frac{\Delta x}{2}}=2 I \sin \frac{k \Delta x}{2}$ so (5.6) can be simplified as follows

$$
\begin{cases}\frac{-2 I}{\Delta t} \sin \frac{\omega \Delta t}{2} \eta+\frac{d e^{I \omega \frac{\Delta t}{2}}}{3 \Delta x} 2 I \sin \frac{k \Delta x}{2}\left(u_{1}+u_{2}+u_{3}\right) & =0 \\ \frac{-2 I}{\Delta t} \sin \frac{\omega \Delta t}{2} u_{i}+\frac{e^{-I \omega \frac{\Delta t}{2}}}{\rho \Delta x} 2 I \sin \frac{k \Delta x}{2} p_{i} & =0 ; i=1,2,3 \\ \frac{-2 I}{\Delta t} \sin \frac{\omega \Delta t}{2} w_{\frac{1}{2}}+\frac{6 g e^{-I \omega \frac{\Delta t}{2}}}{d} \eta-\frac{6 e^{-I \omega \frac{\Delta t}{2}}}{\rho d} p_{1} & =0 \\ \frac{-2 I}{\Delta t} \sin \frac{\omega \Delta t}{2} w_{i+\frac{1}{2}}+\frac{3 e^{-I \omega \frac{\Delta t}{2}}}{\rho d}\left(p_{i}-p_{i+1}\right) & =0 ; i=1,2 \\ \frac{2 I}{\Delta t} \sin \frac{k \Delta x}{2} u_{i}+\frac{3}{d}\left(w_{i-\frac{1}{2}}-w_{i+\frac{1}{2}}\right) & =0 ; i=1,2,3\end{cases}
$$

For $\Delta t \longrightarrow 0$ and $\Delta x \longrightarrow 0$ then $\frac{2}{\Delta t} \sin \frac{\omega \Delta t}{2} \longrightarrow \omega$ and $\frac{2}{\Delta x} \sin \frac{k \Delta x}{2} \longrightarrow k$, so we can arrange (5.7) to a matrix equation below

\begin{tabular}{|c|c|c|c|c|c|c|c|c|c|c|c|}
\hline$-I \omega$ & $\frac{I k d e^{\alpha}}{3}$ & $\frac{I k d e^{\alpha}}{3}$ & $\frac{I k d e^{\alpha}}{3}$ & 0 & 0 & 0 & 0 & 0 & 0 & $\eta$ & \\
\hline 0 & $-I \omega$ & 0 & 0 & 0 & 0 & 0 & $\frac{I k e^{-\alpha}}{\rho}$ & 0 & 0 & $u_{1}$ & \\
\hline 0 & 0 & $-I \omega$ & 0 & 0 & 0 & 0 & 0 & $\frac{I k e^{-\alpha}}{\rho}$ & 0 & $u_{2}$ & \\
\hline 0 & 0 & 0 & $-I \omega$ & 0 & 0 & 0 & 0 & 0 & $\frac{I k e^{-\alpha}}{\rho}$ & $u_{3}$ & \\
\hline$\frac{6 g e^{-\alpha}}{d}$ & 0 & 0 & 0 & $-I \omega$ & 0 & 0 & $\frac{-6 e^{-\alpha}}{d \rho}$ & 0 & 0 & $w_{\frac{1}{2}}$ & \\
\hline 0 & 0 & 0 & 0 & 0 & $-I \omega$ & 0 & $\frac{3 e^{-\alpha}}{d \rho}$ & $\frac{-3 e^{-\alpha}}{d \rho}$ & 0 & $w_{\frac{3}{2}}$ & \\
\hline 0 & 0 & 0 & 0 & 0 & 0 & $-I \omega$ & 0 & $\frac{3 e^{-\alpha}}{d \rho}$ & $\frac{-3 e^{-\alpha}}{d \rho}$ & $w_{\frac{5}{2}}$ & \\
\hline 0 & $I k$ & 0 & 0 & $\frac{3}{d}$ & $\frac{-3}{d}$ & 0 & 0 & 0 & 0 & $p_{1}$ & \\
\hline 0 & 0 & $I k$ & 0 & 0 & $\frac{3}{d}$ & $\frac{-3}{d}$ & 0 & 0 & 0 & $p_{2}$ & \\
\hline 0 & 0 & 0 & $I k$ & 0 & 0 & $\frac{3}{d}$ & 0 & 0 & 0 & $p_{3}$ & \\
\hline
\end{tabular}

with $\left(\alpha=I \omega \frac{\Delta t}{2}\right)$.

Write the matrix equation above as $\mathbf{M U}=\mathbf{0}$. So that the matrix equation has a non 
trivial solution then determinant of $\mathbf{M}$ must be zero, i.e.

$$
\begin{array}{r}
-\frac{\omega^{2} e^{-3 \alpha}}{d^{6} \rho^{3}}\left(6 g k^{6} d^{5}+216 g k^{4} d^{3}+1458 g k^{2} d-(k d)^{6} \omega^{2}-54(k d)^{4} \omega^{2}-729(k d)^{2} \omega^{2}-1458 \omega^{2}\right)=0 \\
\Leftrightarrow g k^{2} d\left(6(k d)^{4}+216(k d)^{2}+1458\right)-\omega^{2}\left((k d)^{6}+54(k d)^{4}+729(k d)^{2}+1458\right)=0
\end{array}
$$

Solving the last equation for $\omega$ and writing the solution as a functions of $k$ and $d$ to obtain the following numerical dispersion relation

$$
\omega^{2}=\frac{g k^{2} d\left(6(k d)^{4}+216(k d)^{2}+1458\right)}{(k d)^{6}+54(k d)^{4}+729(k d)^{2}+1458}
$$

